\title{
NEUES, WISSENSWERTES
}

\section{COVID-19: Wirksamkeit integrativer Medizin?}

Das Auftreten des Virus SARS-CoV-2, welches die als COVID-19 (Coronavirus Disease-19) bezeichnete Atemwegserkrankung verursacht, soll in China bereits im September 2019 begonnen haben [1,2] und führte schließlich im April 2020 zu einer Pandemie [3]., ,Zwischen dem 13. September und 7. Dezember begann sich das Coronavirus dann zu verbreiten - immer mehr Menschen wurden infiziert", so Peter Forster, Erstautor einer im Proc. Natl. Acad. Sci. USA (IF 9.58) veröffentlichten Arbeit [1,2].

In Wuhan wurden insgesamt 16 provisorische Krankenhäuser gebaut, aber nur eines mit dem Namen Jiangxia war hauptsächlich auf TCM spezialisiert. 564 PatientInnen mit leichten Symptomen von COVID-19 wurden dort unter anderem mit traditionellen Kräutern und anderen Methoden wie beispielsweise Akupunktur behandelt $[4,5]$.

Während des COVID-19-Ausbruchs schickte China über 3.200 Personen vom medizinischen Personal aus seinen 600 TCM-Krankenhäusern in die Provinz Hubei. Die staatliche Verwaltung fur TCM (SATCM) entsandte außerdem ein Expertenteam unter der Leitung der drei Professoren Zhang Boli (Abb. 1), Huang Luqi und Tong Xiaolin zum damaligen Zentrum der Epidemie.

Die Kombination aus TCM und westlicher Medizin half wirksam, Fieber, trockenen Husten, Kopfschmerzen, Halsschmerzen, Müdigkeit, Durchfall und andere Symptome von PatientInnen mit leichtem COVID-19 zu lindern. Insbesondere trat der Übergang von leichtmittelschweren zu schwerkritischen Phasen von COVID-19 bei 10\% der PatientInnen auf, die nur westliche Medizin erhielten, verglichen mit nur 4,1\% der PatientInnen, die sowohl in die westliche als auch in die TCMTherapie integriert waren. Diese Daten wurden vom Leiter Professor Zhang Boli bestätigt [6].

China möchte seine TCM-Erfahrungen während der COVID-19-Pandemie nun mit anderen Ländern weltweit teilen. Es wird interessant sein, ob die globale Krise zu einerTCM-Standardisierung für solche Pandemien führen wird oder ob die TCM als wertvolle Medizin hauptsächlich asiatischen Ländern vorbehalten sein wird.

Danksagung: Der Beitrag wurde in erweiterter Form in englischer Sprache als Editorial in „Medical Acupuncture“ im April 2020 veröffentlicht [3].

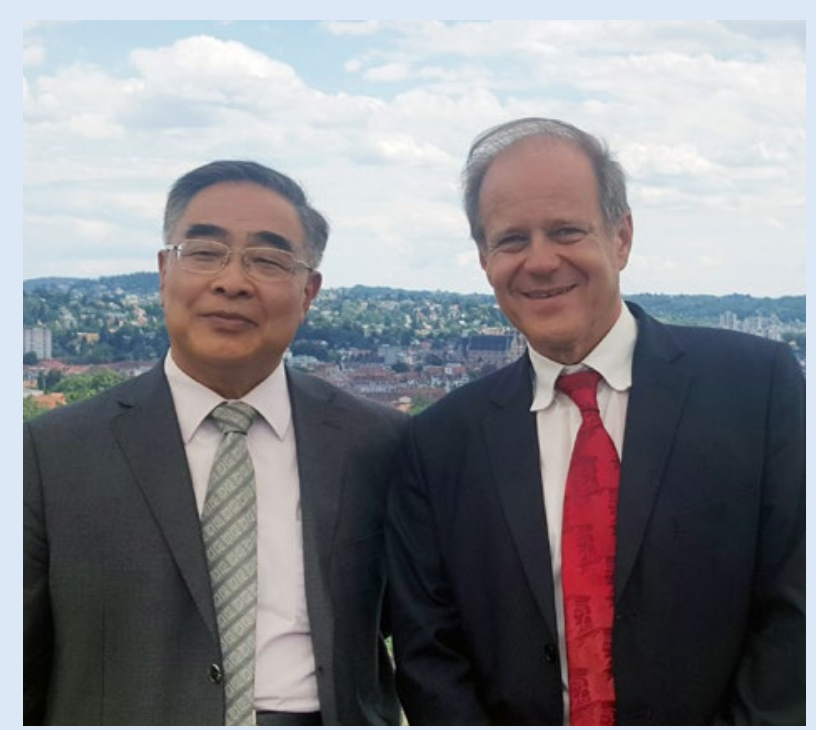

Abb. 1: Professor and „Academician" Zhang Boli mit Professor Gerhard Litscher anlässlich eines Besuchs an der Medizinischen Universität Graz. Prof. Zhang Boli ist ehemaliger Präsident der Chinesischen Akademie für Chinesische Medizin in Peking und ehemaliger Präsident der Tianjin Universität für TCM. Er leitete das TCM Expertenteam während der COVID-19-Krise in Wuhan.

\section{Literatur}

[1] Forster P, Forster L, Renfrew C, Forster M. Phylogenetic network analysis of SARS-CoV-2 genomes. Proc Natl Acad Sci U S A 2020; 117: 9241-9243. doi:10.1073/ pnas.2004999117.

[2] Coronavirus: Erster Fall schon am 13. September? https:// www.nau.ch/amp/news/ausland/coronavirus-mehr-als150000-todesopfer-weltweit-65694944. Stand: 02.06.2020.

[3] Litscher G. Effectiveness of integrated medicine in COVID-19? Editorial. Med Acupunct 2020. doi:10.1089/ acu.2020.29143.1ch.

[4] Liang FX, Litscher G. COVID-19 (Coronavirus Disease-19): Traditional Chinese Medicine including Acupuncture for Alleviation - A Report from Wuhan, Hubei Province in China. OBM Integr Complement Med 2020; 5: 4.

[5] China Internet Information Center (China.org.cn). Renowned Chinese expert Zhong Nanshan shares ideas on COVID-19 control with foreigners. Xinhua, April 15, 2020. http://www.china.org.cn/china/2020-04/15/content_75936241.htm. Stand: 02.06.2020.

[6] China Internet Information Center (China.org.cn). Zhang Boli: The efficacy of TCM in the fight against COVID-19. http://www.china.org.cn/china/special_coverage/ 2020-04/06/content_75899205.htm. Stand: 02.06.2020. This article was published in Science and Technology Daily in Chinese, and translated by Zhang Liying. 